\title{
Ordering phenomena of spin trimers accompanied by a large geometrical Hall effect
}

\author{
Shang Gao, ${ }^{1, *, \dagger}$ Max Hirschberger $\odot,{ }^{1, *}, *$ Oksana Zaharko, ${ }^{2}$ Taro Nakajima,,${ }^{1}$ Takashi Kurumaji $\odot,{ }^{1,}$ Akiko Kikkawa, \\ Junichi Shiogai, ${ }^{3}$ Atsushi Tsukazaki, ${ }^{3}$ Shojiro Kimura, ${ }^{3}$ Satoshi Awaji $\odot,{ }^{3}$ \\ Yasujiro Taguchi ${ }^{\circ},{ }^{1}$ Taka-hisa Arima, ${ }^{1,4}$ and Yoshinori Tokura ${ }^{1,5}$ \\ ${ }^{1}$ RIKEN Center for Emergent Matter Science, Wako 351-0198, Japan \\ ${ }^{2}$ Laboratory for Neutron Scattering and Imaging, Paul Scherrer Institut, CH-5232 Villigen PSI, Switzerland \\ ${ }^{3}$ Institute for Materials Research, Tohoku University, Sendai 980-8577, Japan \\ ${ }^{4}$ Department of Advanced Materials Science, University of Tokyo, Kashiwa 277-8561, Japan \\ ${ }^{5}$ Department of Applied Physics and Tokyo College, University of Tokyo, Tokyo 113-8656, Japan
}

(Received 14 August 2019; published 27 December 2019)

\begin{abstract}
The wave function of conduction electrons moving in the background of a noncoplanar spin structure can gain a quantal phase - the Berry phase — as if the electrons were moving in a strong fictitious magnetic field. Such an emergent magnetic field effect is approximately proportional to the solid angle subtended by the spin moments on three neighboring spin sites, termed the scalar spin chirality. The entire spin chirality of the crystal, unless macroscopically canceled, causes the geometrical Hall effect of real-space Berry-phase origin, whereas the intrinsic anomalous Hall effect in a conventional metallic ferromagnet is of momentum-space Berry-phase origin induced by relativistic spin-orbit coupling. Here, we report the ordering phenomena of the spin-trimer scalar spin chirality and the consequent large geometrical Hall effect in the breathing kagome lattice compound $\mathrm{Dy}_{3} \mathrm{Ru}_{4} \mathrm{Al}_{12}$, where the $\mathrm{Dy}^{3+}$ moments form noncoplanar spin trimers with local spin chirality. Using neutron diffraction, we show that the local spin chirality of the spin trimers as well as its ferroic/antiferroic orders can be switched by an external magnetic field, accompanying large changes in the geometrical Hall effect. Our finding reveals that systems composed of tunable spin trimers can be a fertile field to explore large emergent electromagnetic responses arising from real-space topological magnetic orders.
\end{abstract}

DOI: 10.1103/PhysRevB.100.241115

Conventional electronic devices are based mainly upon the band dispersions of the conducting electrons and not on their phase factors. However, a better understanding of the topological character of electron bands that has been achieved in recent years is now promising next-generation devices where both the dispersions and the phase factors can be tailored [1,2]. Such a prospect is derived from the fact that the Berry phase, which describes the change of the phase factor for an electron moving adiabatically around a loop in real or reciprocal space, has a direct impact on the electron transport [3]. Specifically, in ferromagnetic systems with broken time-reversal symmetry, it has been established that a nonzero Berry phase over the occupied bands in reciprocal space can be induced by relativistic spin-orbit coupling (SOC), which gives rise to an intrinsic anomalous Hall effect (AHE) with the transverse conductivity $\sigma_{x y}$ proportional to the spin polarization of the conducting electron [4-6].

Nonzero Berry phases and a consequent Hall effect can also arise in a special type of magnet where the magnetic moments form noncoplanar structures. In these magnets, the topologically nontrivial Berry phase is induced not by the

\footnotetext{
*These authors contributed equally to this work.

†Corresponding author: shang.gao@ riken.jp

${ }^{\ddagger}$ Corresponding author: maximilian.hirschberger@ riken.jp

${ }^{\S}$ Present address: Department of Physics, Massachusetts Institute of Technology, Cambridge, Massachusetts 02139, USA.
}

SOC as in the case of the "conventional" AHE, but by nonzero scalar spin chirality $\chi_{i j k}=\boldsymbol{S}_{i} \cdot\left(\boldsymbol{S}_{j} \times \boldsymbol{S}_{k}\right)$, with $\boldsymbol{S}$ denoting the localized spins at vertices $i, j$, and $k$ of a triangle [7-11]. Hereafter we refer to such scalar spin chirality simply as chirality. This chirality-induced Hall effect, here named the geometrical Hall effect (GHE), is readily understood in the real-space picture. Due to the coupling with the localized moments, an electron hopping successively across the triangular sites $i-j-k-i$ will gain a Berry phase that is approximately proportional to the solid angle spanned by the localized moments as if the electrons were circling around a magnetic flux [8].

Despite continuous efforts [12-19], an unambiguous experimental illustration of the correspondence between the noncoplanar triangular spin units and the GHE is still missing. This is partly due to the difficulty in stabilizing commensurate noncoplanar magnetic structures on two-dimensional lattices [14]. For example, on a prototypical kagome lattice, Heisenberg spins normally prefer to order in a coplanar structure due to its amenability to fluctuations [20-22], and the coplanarity is maintained even in a magnetic field [23].

In this Rapid Communication, we show that noncoplanar triangular spin units, or spin trimers [24,25], can be stabilized in the intermetallic compound $\mathrm{Dy}_{3} \mathrm{Ru}_{4} \mathrm{Al}_{12}$, where the magnetic $\mathrm{Dy}^{3+}$ ions constitute a breathing kagome lattice with corner-sharing triangles of two different sizes [see Figs. 1(a) and 1(b)] $[26,27]$. Using neutron diffraction, we find that both the ferroic/antiferroic order and the local chirality of the spin trimers can be tuned by a magnetic field. As long as the entire 
(a)

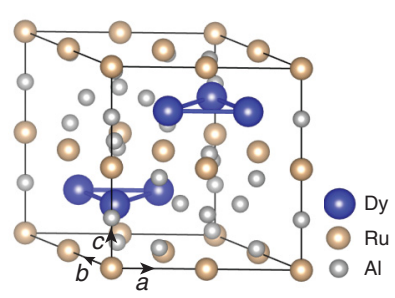

(b)
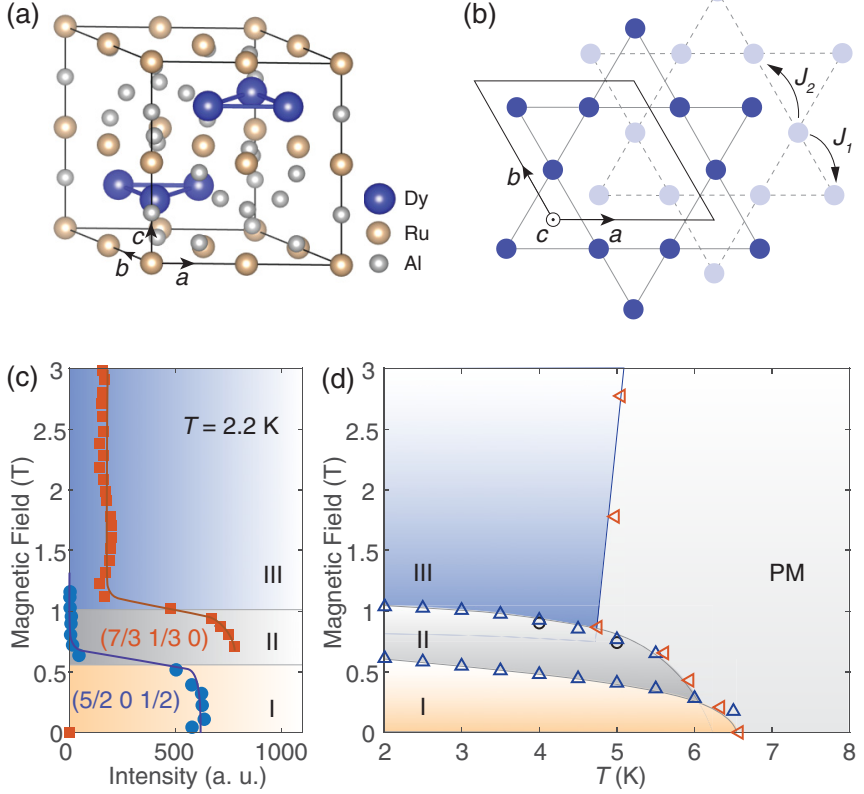

FIG. 1. (a) Crystal structure of $\mathrm{Dy}_{3} \mathrm{Ru}_{4} \mathrm{Al}_{12}$ with space group $P 6_{3} / m m c$. (b) $\mathrm{Dy}^{3+}$ ions on the $6 h$ Wyckoff sites form breathing kagome layers in the $a b$ plane. Dy ${ }^{3+}$ ions with $z=0.25$ and $z=0.75$ are shown by dark and light blue circles, respectively. The nearestneighbor $J_{1}$ and second-neighbor $J_{2}$ bonds are indicated by curved arrows. (c) Field dependence of the neutron diffraction intensities of the magnetic reflections $\left(\frac{5}{2} 0 \frac{1}{2}\right)$ and $\left(\frac{7}{3} \frac{1}{3} 0\right)$ measured at $T=2.2 \mathrm{~K}$. (d) Phase diagram of $\mathrm{Dy}_{3} \mathrm{Ru}_{4} \mathrm{Al}_{12}$ obtained from magnetization (up-pointing triangles) and heat capacity (left-pointing triangles) measurements [28]. PM represents the paramagnetic phase. The effect of the demagnetization field has been corrected [28]. Error bars representing standard deviations are smaller than the symbol size.

scalar spin chirality becomes nonzero, a large GHE emerges in our magnetotransport measurements. The geometrical origin of the observed Hall effect is also confirmed through a semiquantitative comparison between the magnitude of the Hall conductivity and the entire scalar spin chirality.

Our $\mathrm{Dy}_{3} \mathrm{Ru}_{4} \mathrm{Al}_{12}$ single crystals were grown using the Czochralski technique [28]. Neutron diffraction experiments on a single-crystal sample of $\mathrm{Dy}_{3} \mathrm{Ru}_{4} \mathrm{Al}_{12}$ were performed on the thermal-neutron diffractometer ZEBRA at the Swiss Spallation Neutron Source SINQ of the Paul Scherrer Institut PSI. An incoming neutron wavelength of $1.18 \AA$ [ $\mathrm{Ge}(311)$ monochromator] was used for the measurements. Magnetotransport experiments were performed on single crystals with characteristic dimensions of $3.0 \times 0.8 \times 0.15 \mathrm{~mm}^{3}$, where the largest faces were perpendicular to the crystallographic $c$ axis and were aligned perpendicular to the magnetic field. The electric current was applied along the $a^{*}$ axis in reciprocal space. Measurements below $14 \mathrm{~T}$ were performed on the Quantum Design physical property measurement system (PPMS), and measurements up to $24 \mathrm{~T}$ shown in the Supplemental Material were performed at the High Field Laboratory for Superconducting Materials at Tohoku University [28].

At zero field, $\mathrm{Dy}_{3} \mathrm{Ru}_{4} \mathrm{Al}_{12}$ is known to enter a magnetic long-range ordered state with a propagation vector of $\left(\frac{1}{2} 0 \frac{1}{2}\right)$ [26]. However, by applying a magnetic field along the $c$ axis, we found in the present study that the magnetic propagation vector can be shifted from $\boldsymbol{q}_{1}=\left(\frac{1}{2} 0 \frac{1}{2}\right)$ to $\boldsymbol{q}_{2}=\left(\frac{1}{3} \frac{1}{3} 0\right)$. As is shown in Fig. 1(c), our neutron diffraction experiments reveal that at temperature $T=2.2 \mathrm{~K}$, the intensity of the $\left(\frac{5}{2} 0 \frac{1}{2}\right)$ reflection in phase I drops to zero at a field of $\mu_{0} H \approx 0.6 \mathrm{~T}$, while a new reflection emerges at $\left(\frac{7}{3} \frac{1}{3} 0\right)$. The intensity of the $\left(\frac{7}{3} \frac{1}{3} 0\right)$ reflection is not constant, but decreases sharply at $\mu_{0} H \approx 1.2 \mathrm{~T}$ while remaining finite, indicating the appearance of two distinct field-induced phases (II and III), consistent with the magnetic transitions observed by magnetization measurements [26] [see also Fig. 3(a)]. Following the anomalies in the magnetic susceptibility and heat capacity across the phase transitions [28], we map out the $H-T$ phase diagram as presented in Fig. 1(d).

Neutron diffraction data sets were collected in the three phases at $T=2.2 \mathrm{~K}$ to clarify their precise magnetic structures. Figure 2 summarizes our refinement results assuming an equal magnitude for the $\mathrm{Dy}^{3+}$ moments. Details for the dataset refinement can be found in the Supplemental Material [28]. We notice that the magnetic structures in both phases I and II consist of similar spin trimers with nonzero local chirality. As is presented in Fig. 2(d), four spin-trimer configurations are observed in the magnetic structure of phases I and II: The in-plane spin components $S_{a b}$ are all pointing inwards or outwards, and the out-of-plane components $S_{c}$ are aligning uniformly parallel or antiparallel to the $c$ axis. From our refinements, the ratio $\left|S_{c} / S_{a b}\right|$ remains nearly constant at $\sim 2$ in both phases I and II, meaning the magnitude $\chi_{0}$ of the local chirality does not change across the transition between phases I and II. Therefore, the entire chirality is completely determined by the stacking pattern of the trimers. In phase I with $\boldsymbol{q}_{1}=\left(\frac{1}{2} 0 \frac{1}{2}\right)$, the sign of the local chirality is reversed between neighboring unit cells along the $a$ and $c$ axes, implying full cancellation of the local chirality. However, in phase II with $\boldsymbol{q}_{2}=\left(\frac{1}{3} \frac{1}{3} 0\right)$, the signs are arranged in a sequence of $+/+/-$ along the $a$ and $b$ axes, which results in an average chirality per spin-trimer unit of $\chi_{0} / 3$ with $\chi_{0} \approx 0.17$. Here, the chirality $\chi_{0}$ is calculated assuming a unit spin length $|S|=1$. Over the larger second-neighbor triangles, the global chirality also becomes nonzero in phase II, and its magnitude equals $\chi_{0} / 3$, as large as that over the spin-trimer unit.

The individual spin-trimer chirality, as well as the entire chirality averaged over all sites, can be further tuned with a higher magnetic field of $\mu_{0} H>1.2 \mathrm{~T}$, where a new phase (III) is realized. In this phase, most of the magnetic reflections become weaker than those in phase II, and all the $\left(\frac{n}{3} \frac{n}{3} 0\right)$ reflections are extinct. These observations allow us to assign the magnetic structure shown in Fig. 2(c), where the $c$ component of all the $\mathrm{Dy}^{3+}$ moments aligns in the field direction [28]. As shown in Fig. 2(e), the in-plane components of the Dy ${ }^{3+}$ moments change from all-in-all-out to tangential alignment, leading to two twisted trimer configurations with a negative chirality of $-\chi_{0}^{\prime} / 3$ with $\chi_{0}^{\prime} \approx 0.23$. Meanwhile, the twisted trimers also induce toroidal-like correlations over the secondneighbor triangles, where the averaged global chirality per spin-trimer unit amounts to $\chi_{0}^{\prime}$, with a positive sign and an absolute magnitude that is three times as large as that over the nearest-neighbor spin trimers.

The global chirality in phases II and III motivated us to search for the chirality-induced GHE. Figure 3 summarizes 
(a) I: $\boldsymbol{q}=\left(\begin{array}{lll}1 / 2 & 0 & 1 / 2\end{array}\right)$

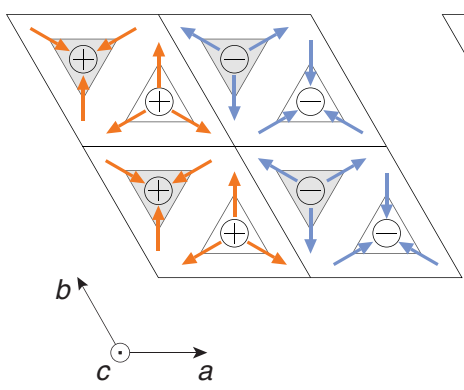

(d)

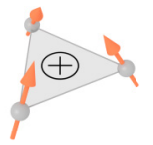

all-in, up

(b)

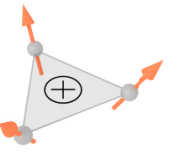

all-out, up

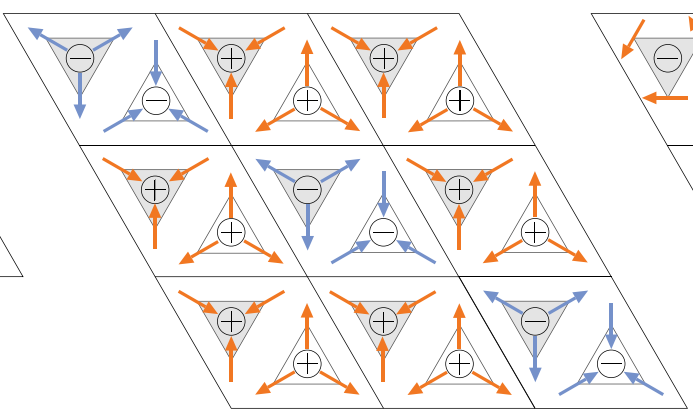

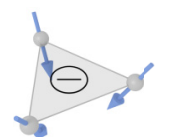

all-in, down

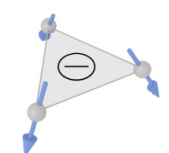

all-out, down (c) III: $\boldsymbol{q}=\left(\begin{array}{ll}1 / 3 & 1 / 30\end{array}\right)$

FIG. 2. (a) Magnetic structure of phase I with $\boldsymbol{q}_{1}=\left(\frac{1}{2} 0 \frac{1}{2}\right)$. Shaded (blank) triangles indicate spin trimers within the $z=0.25(z=0.75)$ layer. Spins with a positive (negative) component along the $c$ direction are shown by the orange (blue) arrows. Signs of the scalar spin chirality over the trimers are indicated at the center of the triangles. (b), (c) Magnetic structures of phases (b) II and (c) III with $\boldsymbol{q}_{2}=\left(\frac{1}{3} \frac{1}{3} 0\right)$. (d) Spin trimers in phases I and II. In these two phases, spin components in the $a b$ plane are either all pointing towards the center of the triangles (all in) or out of the triangles (all out). (e) Twisted and antitwisted spin trimers in phase III where the spin components in the $a b$ plane are parallel to the opposite edge of the triangles. In phases I, II, and III, the refined magnitude of the ordered Dy ${ }^{3+}$ moments are $6.5(5) \mu_{B}, 9.3(5) \mu_{B}$, and $8.9(3) \mu_{B}$, respectively, and the corresponding tilting angle from the $c$ axis is $26(1)^{\circ}, 28(1)^{\circ}$, and $35(1)^{\circ}$.

the results of our transport experiments. The anomalous Hall conductivity $\sigma_{x y}^{\mathrm{A}}$ was obtained by subtracting the normal Hall conductivity $\sigma_{x y}^{\mathrm{N}}$ from the total Hall conductivity $\sigma_{x y}^{\text {tot }}$ with $\sigma_{x y}^{\mathrm{A}}=\sigma_{x y}^{\text {tot }}-\sigma_{x y}^{\mathrm{N}}$ [28]. The total Hall conductivity is related to the longitudinal resistivity $\rho_{x x}$ and the Hall resistivity $\rho_{y x}$ via $\sigma_{x y}^{\text {tot }}=\rho_{y x} /\left(\rho_{x x}^{2}+\rho_{y x}^{2}\right)$. In our definition, the anomalous Hall conductivity $\sigma_{x y}^{\mathrm{A}}$ may involve contributions not only from the conventional SOC-induced Hall term but also from the chirality-induced geometrical Hall term of the present focus.

As is shown in Figs. 3(a) and 3(b), the anomalous Hall conductivity $\sigma_{x y}^{\mathrm{A}}$ becomes nonzero in phases II and III, as is expected for the chirality-induced GHE, leading to a Hall angle $\left(\sigma_{x y}^{\mathrm{A}} / \sigma_{x x}\right)$ as large as $1.5 \times 10^{-2}$ at $3 \mathrm{~T}$ in phase III. The evolution of $\sigma_{x y}^{\mathrm{A}}(H)$ loosely follows the magnetization curve $M(H)$ shown in the same panels. The successive steplike increases in $\sigma_{x y}^{\mathrm{A}}(H)$ across the phase transitions sharply contrast with the highly nonmonotonous evolution of the longitudinal resistivity [28], indicating the independence of $\sigma_{x y}^{\mathrm{A}}$ on the relaxation time $\tau$ of the conducting electrons and thus excluding the extrinsic skew-scattering mechanism [6,29-31] that obeys $\sigma_{x y}^{\text {skew }} \propto \tau$.

Two other possible scenarios for the observed AHE, including the intrinsic Karplus-Luttinger (momentum-space Berry phase) and the extrinsic side-jump mechanisms, can be excluded as the major origin on the basis of the temperature dependence of $\sigma_{x y}^{\mathrm{A}}$ shown in Figs. 3(c) and 3(d). In both mechanisms, $\sigma_{x y}^{\mathrm{A}}$ is independent of the relaxation time $\tau[6,29]$. However, the magnitude of $\sigma_{x y}^{\mathrm{A}}$ induced through the KarplusLuttinger or the side-jump mechanism is not related to chirality of the magnetic moments and only depends on the total magnetization $M$ along the field direction [6]. As is shown in Fig. 3(d), a sharp increase in $\sigma_{x y}^{\mathrm{A}}(T)$ is observed when the system is cooled through the phase transition at $\sim 5 \mathrm{~K}$ in a magnetic field, while the magnetization $M(T)$ almost stays constant on both sides of the transition. The contrasting behavior of $\sigma_{x y}^{\mathrm{A}}(T)$ and $M(T)$ reveals that the observed AHE is not dominated by the Karplus-Luttinger or the side-jump mechanism and is consistent with its chirality origin.

The relative change of $\sigma_{x y}^{\mathrm{A}}$ between phases II and III can be semiquantitatively understood through the chirality-induced GHE. Assuming the geometrical weight over the $\mathrm{Dy}^{3+}$ trimers and the second-neighbor triangles to be dominant and comparable $[10,11]$, the total chirality $\chi^{\text {tot }}$ of the Dy sublattice can be estimated to be $\sim 2 \chi_{0} / 3$ and $2 \chi_{0}^{\prime} / 3$ in phases II and III, respectively, with $\chi_{0}^{\prime} / \chi_{0} \approx 1.4$. Meanwhile, due to the perturbative nature of the couplings between the conducting electrons and the $\mathrm{Dy}^{3+}$ moments, the spin polarization $p$ of the conducting electrons should be proportional to the net magnetization, with $M^{\mu_{0} H=2 \mathrm{~T}} / M^{\mu_{0} H=0.7 \mathrm{~T}} \approx 2.7$. Given that $\sigma_{x y}^{\mathrm{A}} \propto p \chi_{\mathrm{tot}}$, the ratio of the chirality-induced anomalous Hall conductivity in phases III and II is expected to be $\sim 3.8$, which is close to our observation in Fig. 3(a).

Compared to the skyrmion lattice characterized by a topological Hall effect related to the winding number of the spin texture [32-36], the magnetic structures in phases II and III of $\mathrm{Dy}_{3} \mathrm{Ru}_{4} \mathrm{Al}_{12}$ have a much shorter periodicity, where a magnetic unit cell consists only of $3 \times 3$ unit cells. According to theoretical calculations [11], when the couplings between the conducting electrons and local moments are weak as in $\mathrm{Dy}_{3} \mathrm{Ru}_{4} \mathrm{Al}_{12}$, the two Berry-phase scenarios in reciprocal and real spaces can be equivalent. Therefore, $\mathrm{Dy}_{3} \mathrm{Ru}_{4} \mathrm{Al}_{12}$ might be viewed as an intermediate system that bridges the magnetic skyrmion lattice with a long periodicity of tens of nanometers [34] and the prototypical kagome model with a minimal periodicity of one single unit cell [8], and might help 

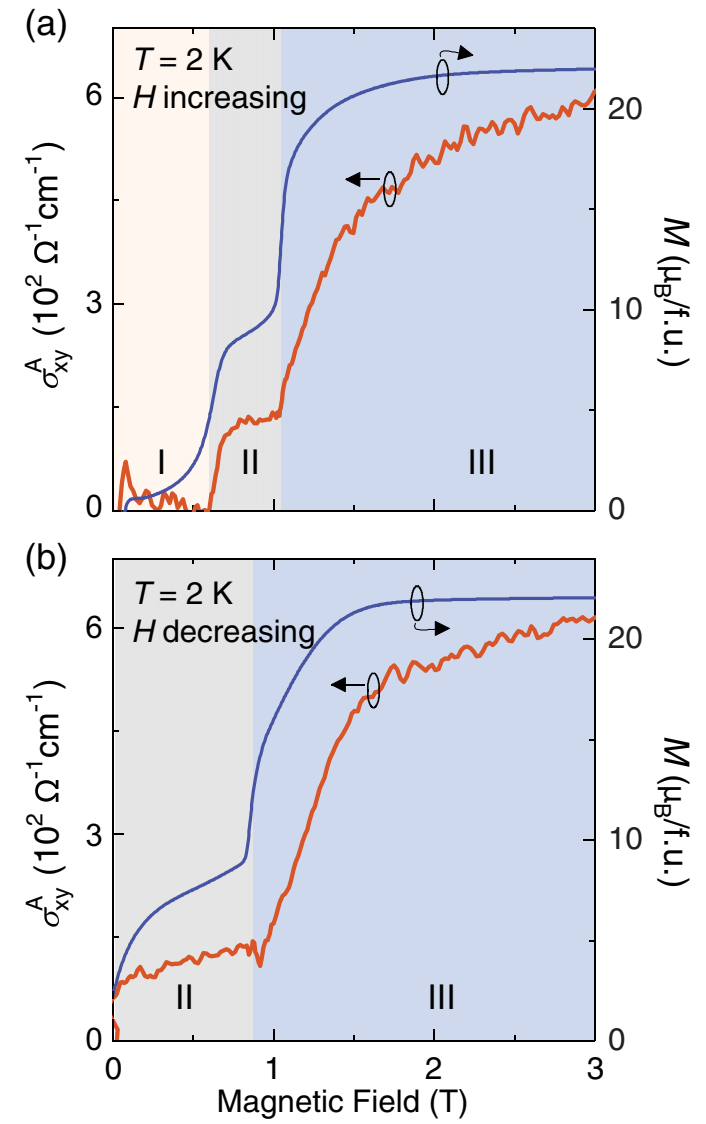
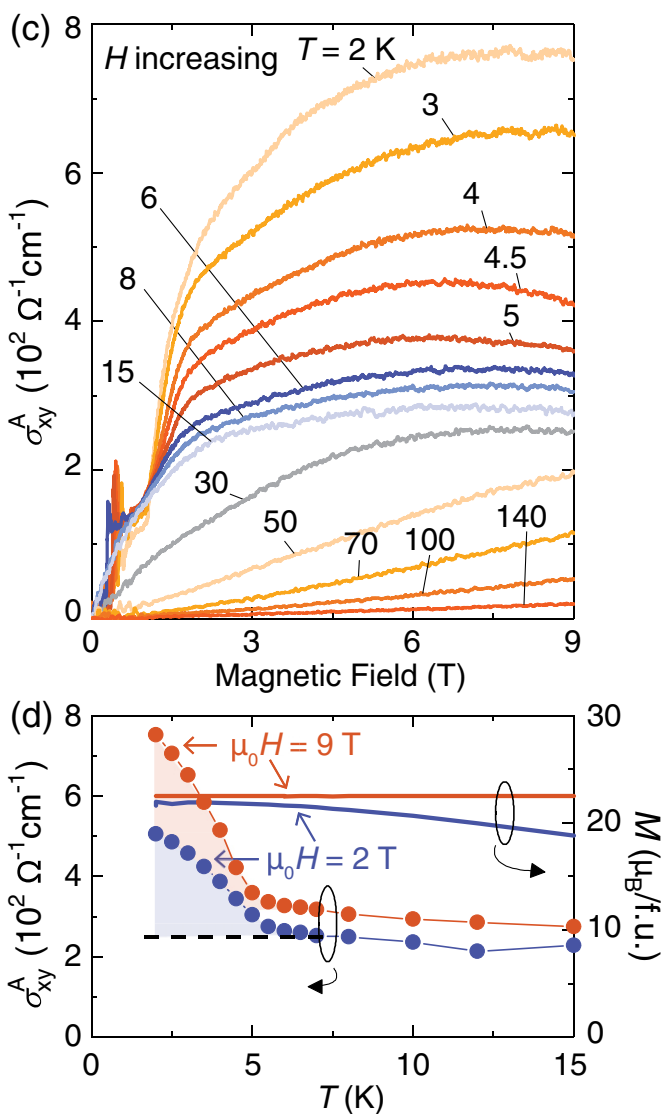

FIG. 3. (a), (b) Anomalous Hall conductivity $\sigma_{x y}^{\mathrm{A}}$ measured at $2 \mathrm{~K}$ as a function of (a) increasing and (b) decreasing magnetic fields. The corresponding field dependence of magnetization $M(H)$ is shown for comparison. Different phases are indicated by color shading, and a large hysteresis for increasing and decreasing fields is observed at the phase boundaries. Note $\sigma_{x y}^{\mathrm{A}}=0$ in phase I, where scalar spin chirality is absent, and a sharp increase of $\sigma_{x y}^{\mathrm{A}}$ at the boundary between phases II and III. (c) Full view of $\sigma_{x y}^{\mathrm{A}}$ data, including high fields and temperatures. (d) Temperature dependence of $\sigma_{x y}^{\mathrm{A}}$ (solid circles) and magnetization (solid lines) measured in external magnetic fields of $2 \mathrm{~T}$ (blue) and $9 \mathrm{~T}$ (red). A sharp increase in $\sigma_{x y}^{\mathrm{A}}(T)$ when entering phase III below $\sim 5 \mathrm{~K}$ is in strong contrast to the behavior of $M(T)$ in the same field, evidencing the onset of nonzero scalar spin chirality at $T \sim 5 \mathrm{~K}$. The effect of the demagnetization field has been corrected [28].

clarify the equivalence between the two Berry-phase scenarios for the GHE in reciprocal and real spaces.

Due to the strong SOC on the Ru ions, we do not completely rule out the role of SOC in our observed GHE. The SOC may join the scalar spin chirality in enhancing the transfer of the Berry curvature. Recent investigations on $\mathrm{Mn}_{3} \mathrm{Sn}$ and $\mathrm{Mn}_{3} \mathrm{Ge}$ reveal that the SOC is able to induce an AHE even in antiferromagnets with coplanar magnetic structures [37-40]. It will be interesting to clarify the role of the SOC and explore its possible interplay with the scalar spin chirality in $\mathrm{Dy}_{3} \mathrm{Ru}_{4} \mathrm{Al}_{12}$.

In summary, our neutron diffraction experiments reveal the existence of spin trimers in the breathing kagome lattice compound $\mathrm{Dy}_{3} \mathrm{Ru}_{4} \mathrm{Al}_{12}$, where both the stacking order and the local scalar spin chirality of the trimers can be tuned by a magnetic field. In phases with nonzero entire scalar spin chirality, a large GHE is observed in our magnetotransport ex- periment. Our works provide an unambiguous illustration for the chirality-induced GHE, and reveal that systems composed of tunable spin trimers can exhibit large emergent electromagnetic response due to couplings between the conduction electrons and the localized magnetic moments.

We acknowledge helpful discussions with H. Ishizuka, N. Nagaosa, N. Gauthier, B. Normand, O. Benton, C. L. Zhang, and C. Mudry. Our neutron diffraction experiments were performed at the Swiss Spallation Neutron Source SINQ, Paul Scherrer Insitut PSI, Villigen, Switzerland. Our transport experiment in high magnetic fields up to $24 \mathrm{~T}$ were measured at the High Field Laboratory for Superconducting Materials at Tohoku University. This work was supported in part by JST CREST Grant No. JPMJCR1874 (Japan). M.H. was supported as a JSPS International Research Fellow (Grant No. 18F18804).
[1] X.-L. Qi and S.-C. Zhang, Topological insulators and superconductors, Rev. Mod. Phys. 83, 1057 (2011).
[2] Y. Tokura, M. Kawasaki, and N. Nagaosa, Emergent functions of quantum materials, Nat. Phys. 13, 1056 (2017). 
[3] D. Xiao, M.-C. Chang, and Q. Niu, Berry phase effects on electronic properties, Rev. Mod. Phys. 82, 1959 (2010).

[4] Z. Fang, N. Nagaosa, K. S. Takahashi, A. Asamitsu, R. Mathieu, T. Ogasawara, H. Yamada, M. Kawasaki, Y. Tokura, and K. Terakura, The anomalous Hall effect and magnetic monopoles in momentum space, Science 302, 92 (2003).

[5] Y. Yao, L. Kleinman, A. H. MacDonald, J. Sinova, T. Jungwirth, D.-s. Wang, E. Wang, and Q. Niu, First Principles Calculation of Anomalous Hall Conductivity in Ferromagnetic bcc Fe, Phys. Rev. Lett. 92, 037204 (2004).

[6] N. Nagaosa, J. Sinova, S. Onoda, A. H. MacDonald, and N. P. Ong, Anomalous Hall effect, Rev. Mod. Phys. 82, 1539 (2010).

[7] J. Ye, Y. B. Kim, A. J. Millis, B. I. Shraiman, P. Majumdar, and Z. Tešanović, Berry Phase Theory of the Anomalous Hall Effect: Application to Colossal Magnetoresistance Manganites, Phys. Rev. Lett. 83, 3737 (1999).

[8] K. Ohgushi, S. Murakami, and N. Nagaosa, Spin anisotropy and quantum Hall effect in the kagomé lattice: Chiral spin state based on a ferromagnet, Phys. Rev. B 62, R6065(R) (2000).

[9] R. Shindou and N. Nagaosa, Orbital Ferromagnetism and Anomalous Hall Effect in Antiferromagnets on the Distorted fcc Lattice, Phys. Rev. Lett. 87, 116801 (2001).

[10] G. Tatara and H. Kawamura, Chirality-driven anomalous Hall effect in weak coupling regime, J. Phys. Soc. Jpn. 71, 2613 (2002).

[11] M. Onoda, G. Tatara, and N. Nagaosa, Anomalous Hall effect and skyrmion number in real and momentum spaces, J. Phys. Soc. Jpn. 73, 2624 (2004).

[12] P. Matl, N. P. Ong, Y. F. Yan, Y. Q. Li, D. Studebaker, T. Baum, and G. Doubinina, Hall effect of the colossal magnetoresistance manganite $\mathrm{La}_{1-x} \mathrm{Ca}_{x} \mathrm{MnO}_{3}$, Phys. Rev. B 57, 10248 (1998).

[13] Y. Taguchi, Y. Oohara, H. Yoshizawa, N. Nagaosa, and Y. Tokura, Spin chirality, Berry phase, and anomalous Hall effect in a frustrated ferromagnet, Science 291, 2573 (2001).

[14] D. Grohol, K. Matan, J.-H. Cho, S.-H. Lee, J. W. Lynn, D. G. Nocera, and Y. S. Lee, Spin chirality on a two-dimensional frustrated lattice, Nat. Mater. 4, 323 (2005).

[15] H. Takatsu, S. Yonezawa, S. Fujimoto, and Y. Maeno, Unconventional Anomalous Hall Effect in the Metallic TriangularLattice Magnet $\mathrm{PdCrO}_{2}$, Phys. Rev. Lett. 105, 137201 (2010).

[16] Y. Machida, S. Nakatsuji, S. Onoda, T. Tayama, and T. Sakakibara, Time-reversal symmetry breaking and spontaneous Hall effect without magnetic dipole order, Nature (London) 463, 210 (2010).

[17] Y. Shiomi, M. Mochizuki, Y. Kaneko, and Y. Tokura, Hall Effect of Spin-Chirality Origin in a Triangular-Lattice Helimagnet $\mathrm{Fe}_{1.3}$ Sn, Phys. Rev. Lett. 108, 056601 (2012).

[18] B. Ueland, C. Miclea, Y. Kato, O. Ayala-Valenzuela, R. McDonald, R. Okazaki, P. Tobash, M. Torrez, F. Ronning, R. Movshovich, Z. Fisk, E. Bauer, I. Martin, and J. Thompson, Controllable chirality-induced geometrical Hall effect in a frustrated highly correlated metal, Nat. Commun. 3, 1067 (2012).

[19] C. Sürgers, G. Fischer, P. Winkel, and H. v. Löhneysen, Large topological Hall effect in the non-collinear phase of an antiferromagnet, Nat. Commun. 5, 3400 (2014).

[20] J. N. Reimers and A. J. Berlinsky, Order by disorder in the classical Heisenberg kagomé antiferromagnet, Phys. Rev. B 48, 9539 (1993).
[21] M. Elhajal, B. Canals, and C. Lacroix, Symmetry breaking due to Dzyaloshinsky-Moriya interactions in the kagomé lattice, Phys. Rev. B 66, 014422 (2002).

[22] K. Essafi, O. Benton, and L. D. C. Jaubert, Generic nearestneighbor kagome model: XYZ and Dzyaloshinskii-Moriya couplings with comparison to the pyrochlore-lattice case, Phys. Rev. B 96, 205126 (2017).

[23] M. E. Zhitomirsky, Field-Induced Transitions in a Kagomé Antiferromagnet, Phys. Rev. Lett. 88, 057204 (2002).

[24] S. Nakamura, N. Kabeya, M. Kobayashi, K. Araki, K. Katoh, and A. Ochiai, Spin trimer formation in the metallic compound $\mathrm{Gd}_{3} \mathrm{Ru}_{4} \mathrm{Al}_{12}$ with a distorted kagome lattice structure, Phys. Rev. B 98, 054410 (2018).

[25] T. Matsumura, Y. Ozono, S. Nakamura, N. Kabeya, and A. Ochiai, Helical ordering of spin trimers in a distorted kagome lattice of $\mathrm{Gd}_{3} \mathrm{Ru}_{4} \mathrm{Al}_{12}$ studied by resonant x-ray diffraction, J. Phys. Soc. Jpn. 88, 023704 (2019).

[26] D. I. Gorbunov, M. S. Henriques, A. V. Andreev, A. Gukasov, V. Petříček, N. V. Baranov, Y. Skourski, V. Eigner, M. Paukov, J. Prokleška, and A. P. Gonçalves, Electronic properties of a distorted kagome lattice antiferromagnet $\mathrm{Dy}_{3} \mathrm{Ru}_{4} \mathrm{Al}_{12}$, Phys. Rev. B 90, 094405 (2014).

[27] I. Ishii, T. Mizuno, K. Takezawa, S. Kumano, Y. Kawamoto, T. Suzuki, D. I. Gorbunov, M. S. Henriques, and A. V. Andreev, Magnetic-field-induced quadrupolar ordering and the crystal electric field effect in the distorted kagome lattice antiferromagnet $\mathrm{Dy}_{3} \mathrm{Ru}_{4} \mathrm{Al}_{12}$, Phys. Rev. B 97, 235130 (2018).

[28] See Supplemental Material at http://link.aps.org/supplemental/ 10.1103/PhysRevB.100.241115 for details on the sample preparation and characterizations, refinements of the neutron diffraction data sets, magnetotransport experiments, and discussions on the phase transition and the spin-trimer stability, which includes Refs. [41-60].

[29] S. Onoda, N. Sugimoto, and N. Nagaosa, Quantum transport theory of anomalous electric, thermoelectric, and thermal Hall effects in ferromagnets, Phys. Rev. B 77, 165103 (2008).

[30] N. Manyala, Y. Sidis, J. F. DiTusa, G. Aeppli, D. P. Young, and Z. Fisk, Large anomalous Hall effect in a silicon-based magnetic semiconductor, Nat. Mater. 3, 255 (2004).

[31] T. Miyasato, N. Abe, T. Fujii, A. Asamitsu, S. Onoda, Y. Onose, N. Nagaosa, and Y. Tokura, Crossover Behavior of the Anomalous Hall Effect and Anomalous Nernst Effect in Itinerant Ferromagnets, Phys. Rev. Lett. 99, 086602 (2007).

[32] M. Lee, W. Kang, Y. Onose, Y. Tokura, and N. P. Ong, Unusual Hall Effect Anomaly in MnSi Under Pressure, Phys. Rev. Lett. 102, 186601 (2009).

[33] R. Ritz, M. Halder, M. Wagner, C. Franz, A. Bauer, and C. Pfleiderer, Formation of a topological non-Fermi liquid in MnSi, Nature (London) 497, 231 (2013).

[34] N. Nagaosa and Y. Tokura, Topological properties and dynamics of magnetic skyrmions, Nat. Nano 8, 899 (2013).

[35] T. Kurumaji, T. Nakajima, M. Hirschberger, A. Kikkawa, Y. Yamasaki, H. Sagayama, H. Nakao, Y. Taguchi, T.-h. Arima, and Y. Tokura, Skyrmion lattice with a giant topological Hall effect in a frustrated triangular-lattice magnet, Science 365, 914 (2019).

[36] M. Hirschberger, T. Nakajima, S. Gao, L. Peng, A. Kikkawa, T. Kurumaji, M. Kriener, Y. Yamasaki, H. Sagayama, H. Nakao, K. Ohishi, K. Kakurai, Y. Taguchi, X. Yu, T.-h. Arima, and Y. 
Tokura, Skyrmion phase and competing magnetic orders on a breathing kagome lattice, arXiv:1812.02553.

[37] H. Chen, Q. Niu, and A. H. MacDonald, Anomalous Hall Effect Arising from Noncollinear Antiferromagnetism, Phys. Rev. Lett. 112, 017205 (2014).

[38] S. Nakatsuji, N. Kiyohara, and T. Higo, Large anomalous Hall effect in a non-collinear antiferromagnet at room temperature, Nature (London) 527, 212 (2015).

[39] A. K. Nayak, J. E. Fischer, Y. Sun, B. Yan, J. Karel, A. C. Komarek, C. Shekhar, N. Kumar, W. Schnelle, J. Kübler, C. Felser, and S. S. P. Parkin, Large anomalous Hall effect driven by a nonvanishing Berry curvature in the noncollinear antiferromagnet $\mathrm{Mn}_{3} \mathrm{Ge}$, Sci. Adv. 2, e1501870 (2016).

[40] J. Liu and L. Balents, Anomalous Hall Effect and Topological Defects in Antiferromagnetic Weyl Semimetals: $\mathrm{Mn}_{3} \mathrm{Sn} / \mathrm{Ge}$, Phys. Rev. Lett. 119, 087202 (2017).

[41] F. Izumi and K. Momma, Three-dimensional visualization in powder diffraction, Solid State Phenom. 130, 15 (2007).

[42] J. A. Osborn, Demagnetizing factors of the general ellipsoid, Phys. Rev. 67, 351 (1945).

[43] V. Petř́íček, M. Dušek, and L. Palatinus, Crystallographic computing system JANA2006: General features, Z. Kristallogr. 229, 345 (2014).

[44] J. Rodrguez-Carvajal, Recent advances in magnetic structure determination by neutron powder diffraction, Physica B 192, 55 (1993).

[45] J. Perez-Mato, S. Gallego, E. Tasci, L. Elcoro, G. de la Flor, and M. Aroyo, Symmetry-based computational tools for magnetic crystallography, Annu. Rev. Mater. Res. 45, 217 (2015).

[46] Bilbao crystallographic server, http://www.cryst.ehu.es.

[47] B. Metcalf, Ground state spin orderings of the triangular Ising model with the nearest and next nearest neighbor interaction, Phys. Lett. A 46, 325 (1974).

[48] W. Ge, C. Michioka, H. Ohta, and K. Yoshimura, Physical properties of the layered compounds $R E_{3} \mathrm{Ru}_{4} \mathrm{Al}_{12}(R E=\mathrm{La}-\mathrm{Nd})$, Solid State Commun. 195, 1 (2014).

[49] M. S. Henriques, D. I. Gorbunov, A. V. Andreev, X. Fabrèges, A. Gukasov, M. Uhlarz, V. Petříček, B. Ouladdiaf, and J.
Wosnitza, Complex magnetic order in the kagome ferromagnet $\mathrm{Pr}_{3} \mathrm{Ru}_{4} \mathrm{Al}_{12}$, Phys. Rev. B 97, 014431 (2018).

[50] D. I. Gorbunov, M. S. Henriques, A. V. Andreev, V. Eigner, A. Gukasov, X. Fabrèges, Y. Skourski, V. Petříček, and J. Wosnitza, Magnetic anisotropy and reduced neodymium magnetic moments in $\mathrm{Nd}_{3} \mathrm{Ru}_{4} \mathrm{Al}_{12}$, Phys. Rev. B 93, 024407 (2016).

[51] S. Rayaprol, A. Hoser, K. K. Iyer, S. K. Upadhyay, and E. Sampathkumaran, Neutron diffraction study of a metallic kagome lattice, $\mathrm{Tb}_{3} \mathrm{Ru}_{4} \mathrm{Al}_{12}$, J. Magn. Magn. Mater. 477, 83 (2019).

[52] D. I. Gorbunov, T. Nomura, I. Ishii, M. S. Henriques, A. V. Andreev, M. Doerr, T. Stöter, T. Suzuki, S. Zherlitsyn, and J. Wosnitza, Crystal-field effects in the kagome antiferromagnet $\mathrm{Ho}_{3} \mathrm{Ru}_{4} \mathrm{Al}_{12}$, Phys. Rev. B 97, 184412 (2018).

[53] S. K. Upadhyay, K. K. Iyer, and E. V. Sampathkumaran, Magnetic behavior of metallic kagome lattices, $\mathrm{Tb}_{3} \mathrm{Ru}_{4} \mathrm{Al}_{12}$ and $\mathrm{Er}_{3} \mathrm{Ru}_{4} \mathrm{Al}_{12}$, J. Phys.: Condens. Matter 29, 325601 (2017).

[54] S. Nakamura, S. Toyoshima, N. Kabeya, K. Katoh, T. Nojima, and A. Ochiai, Low-temperature properties of the $S=1 / 2$ spin system $\mathrm{Yb}_{3} \mathrm{Ru}_{4} \mathrm{Al}_{12}$ with a distorted kagome lattice structure, Phys. Rev. B 91, 214426 (2015).

[55] J. Jensen and A. R. Mackintosh, Rare Earth Magnetism (Clarendon Press, Oxford, UK, 1991).

[56] Y.-P. Huang, G. Chen, and M. Hermele, Quantum Spin Ices and Topological Phases from Dipolar-Octupolar Doublets on the Pyrochlore Lattice, Phys. Rev. Lett. 112, 167203 (2014).

[57] T. Fennell, S. T. Bramwell, D. F. McMorrow, P. Manuel, and A. R. Wildes, Pinch points and Kasteleyn transitions in kagome ice, Nat. Phys. 3, 566 (2007).

[58] S. Awaji, K. Watanabe, H. Oguro, H. Miyazaki, S. Hanai, T. Tosaka, and S. Ioka, First performance test of a 25 T cryogenfree superconducting magnet, Supercond. Sci. Technol. 30, 065001 (2017).

[59] J. Xiong, Y. Luo, Y.H. Khoo, S. Jia, R. J. Cava, and N. P. Ong, High-field Shubnikov-de Haas oscillations in the topological insulator $\mathrm{Bi}_{2} \mathrm{Te}_{2} \mathrm{Se}$, Phys. Rev. B 86, 045314 (2012).

[60] L. M. Roth and P. N. Argyres, in Semiconductors and Semimetals, edited by R. K. Williardson and A. C. Beer (Academic, New York, 1966), Vol. 1, Chap. 6. 